Research in Mathematics Education

Vol. 11, No. 1, March 2009, pp.75-76

\title{
Towards a Constructionist Approach to Mathematical Generalisation
}

\author{
Eirini Geraniou, Manolis Mavrikis, Celia Hoyles and Richard Noss \\ London Knowledge Lab, Institute of Education, University of London, UK
}

$\underline{\text { e.geraniou, }} \underline{\text { m.mavrikis, }} \underline{\text { c.hoyles, }} \underline{\text { r.noss }\} @ \text { ioe.ac.uk }}$

Accommodating the idea of mathematical generalisation, recognising and analysing pattern and articulating structure, especially expressed in algebraic forms, are key aspects of mathematical thinking and have long been elusive ideas for students. Although students are often able to identify and predict patterns (Mason 2002), they are unable to articulate a general pattern or relationship in natural language or, even more demanding, in algebraic symbolism (Hoyles and Küchemann 2002). What is required therefore, is to design situations that are rich in the construction and analysis of patterns, and provide both a rationale and computational support for expressing generality.

There is evidence that a powerful approach for this kind of interaction is to put students in situations where they can build their own models (Noss and Hoyles 1996). Such a constructionist pedagogical approach supports students' efforts through the construction of their own mathematical models and aims to help them see the general in the particular by construction and exploration.

Our aim in the Migen project* is to design, build and evaluate a pedagogical and technical environment for improving 11-14 year-old students' learning of mathematical generalisation. This system comprises a microworld that attempts to support students in their reasoning and problem-solving of generalisation tasks. Two further components will provide personalised support adapted to students' construction processes and foster and sustain an effective online learning community by advising learners and teachers on constructions they could view, compare, critique and build upon.

The focus of this paper is on a prototype of the microworld (ShapeBuilder), which has led us to radically redesign our system and facilitated the evaluation of our pedagogical and technical approach. An iterative design process was followed, interleaving software development phases with small-scale pilot studies with year 6 and 7 students. We have also integrated feedback from teachers and teacher educators. This co-design with teachers is critical since trying to match learning environments to teachers' goals and expectations for students' learning is of great importance.

Throughout these pilot studies, we focused on the pond-tiling activity, a typical generalisation activity in the British National Curriculum, which could be described simply as: "Given a rectangular pond with an integer length and breadth, how many $1 \mathrm{x} 1$ tiles are needed to surround it?". We have focused on this activity both because we feel we have created a solution space that exploits computational power, and it naturally lends itself to a variety of different representations, which could be described with the use of different yet equivalent expressions for the number of tiles required. Students can explore their own 
solutions, and compare them with others', raising the necessity (or at least encouragement) to represent the relationships semi-formally.

A preliminary data analysis has shown that although some students find it difficult to shift from specific examples to general expressions, the constructionist approach allows them to follow a number of more approachable and achievable steps. Furthermore, the value of the teachers' and researchers' interventions during students' interactions with ShapeBuilder was identified in the data. An example of such an intervention was the "messing-up" strategy (Hoyles and Healy, 1999) that research in Dynamic Geometry Environments has shown to be highly effective. By messing-up their construction by changing a shape's attribute, students could come to see the lack of generality in their constructions, and, more importantly, a rationale for generality. This led naturally to the development of a general rule for the number of surrounding tiles. An important feature of the system, that further assisted students' efforts to find such a rule, is the use of "icon-variables", which are iconic representations of an object's attribute (e.g. length of a rectangle). This design feature can be used as an intermediate step that scaffolds the use of standard, algebraic-style variables and holds significant potential to lead to generalised thinking.

Finally, the teachers' and students' feedback was critical in providing us with an insight into the students' thinking strategies and are the stepping stones for the development of our system as well as further investigation of its typical classroom deployment.

*This ongoing study is funded by the ESRC/EPSRC/TEL, Award Res-139-25-0381.

\section{References}

Hoyles, C., and L. Healy. 1999. Visual and Symbolic Reasoning in Mathematics: Making Connections with Computers. Mathematical Thinking and Learning 1: 59-84.

Hoyles, C. and D. Küchemann. 2002. Students' Understanding of Logical Implication. Educational Studies in Mathematics, 51, 3: 193-223.

Mason, J. H. 2002. Generalisation and Algebra: Exploiting Children's Powers. In Aspects of Teaching Secondary Mathematics: Perspectives on Practice, ed. L. Haggarty, 105-120. Routledge Falmer: London.

Noss, R. and C. Hoyles, 1996. Windows on Mathematical Meanings: Learning Cultures and Computers. Dordrecht: Kluwer. 